\title{
The Flow Pattern in Single and Multiple Submerged Channels with Gas Evolution at the Electrodes
}

\author{
A. Alexiadis, ${ }^{1}$ M. P. Dudukovic, ${ }^{2}$ P. Ramachandran, ${ }^{2}$ A. Cornell, ${ }^{1}$ \\ J. Wanngård, ${ }^{3}$ and A. Bokkers ${ }^{4}$ \\ ${ }^{1}$ Department of Chemical Engineering and Technology, Applied Electrochemistry, Royal Institute of Technology, KTH, \\ 10044 Stockholm, Sweden \\ ${ }^{2}$ Department of Energy, Environmental and Chemical Engineering, Washington University in St. Louis, One Brookings Drive, \\ Campus Box 1180, St. Louis, MO 63130, USA \\ ${ }^{3}$ Process R\&D, Technology and Engineering, Eka Chemicals, 44580 Bohus, Sweden \\ ${ }^{4}$ Research, Development \& Innovation, Akzo Nobel Chemicals bv, Zutphenseweg 10, P.O. Box 10, 7400 AA Deventer, The Netherlands
}

Correspondence should be addressed to A. Alexiadis, alessioa@kth.se

Received 22 November 2011; Accepted 30 January 2012

Academic Editor: Sreepriya Vedantam

Copyright ( 2012 A. Alexiadis et al. This is an open access article distributed under the Creative Commons Attribution License, which permits unrestricted use, distribution, and reproduction in any medium, provided the original work is properly cited.

\begin{abstract}
We show that the gas-liquid flow pattern in a single gas-evolving electrochemical channel can be remarkably different from the flow pattern in multiple submerged gas-evolving electrochemical channels. This is due to the fact that in a single channel there is a higher accumulation of small bubbles and these can considerably affect the liquid velocity pattern which in turn may affect the performance of a cell. Since experimental work is often carried out in single channels, while industrial applications almost always involve multiple channels, this study provides insight into the factors that affect the flow pattern in each situation and establishes the basis for relating the behavior of single-and multiple-channel devices.
\end{abstract}

\section{Introduction}

Many industrial processes are based on electrochemistry and it is anticipated that, if their efficiency can be improved, they will find increased use in new green technologies. Often, however, high power consumption is associated with these processes and most of the production cost is due to electrical energy. Thus, every advance in optimization and improvement of these processes can lead to significant energy conservation and cost reduction. This provides the motivation for many experimental [1-3] and theoretical [4-6] studies in the field. In many cases (e.g., chlorate production, hypochlorite generation, or perchlorate electrolysis) the industrial scale electrochemical cell consists of a series of channels immersed in a bath of electrolyte. A gas (e.g., hydrogen or oxygen), which is the byproduct of the reaction, is often generated at the electrode surfaces. A generic representation of such industrial scale process is sketched in Figure 1 and contains a series (generally of the order of hundreds) of parallel channels (gaps) between electrode plates immersed in a large container (reactor) that contains the electrolytes. The electrodes are the walls of the channels in the reactor. Electrochemically evolved gas is produced at the electrodes in the dissolved state; small bubble nucleation starts at the imperfections of the electrode surface and then these bubbles depart into the surrounding, highly supersaturated, electrolyte $[7,8]$. The generated bubbles are responsible for the gas-lift effect that promotes global circulation of the liquid in the cell. They also enhance local mixing that helps in continuously refreshing the electrolyte in the vicinity of the electrodes.

In contrast, in the laboratory, experiments are often carried out in a single-channel configuration. The implicit assumption is that, if the single-channel is geometrically similar to the ones in a multiple-channel cell, the observed variables of interest will be approximately the same as in all the channels of the larger system. We find that this assumption, under circumstances frequently encountered in practice, is not necessarily valid and the velocity patterns in the single-channel and in the multichannel configuration 


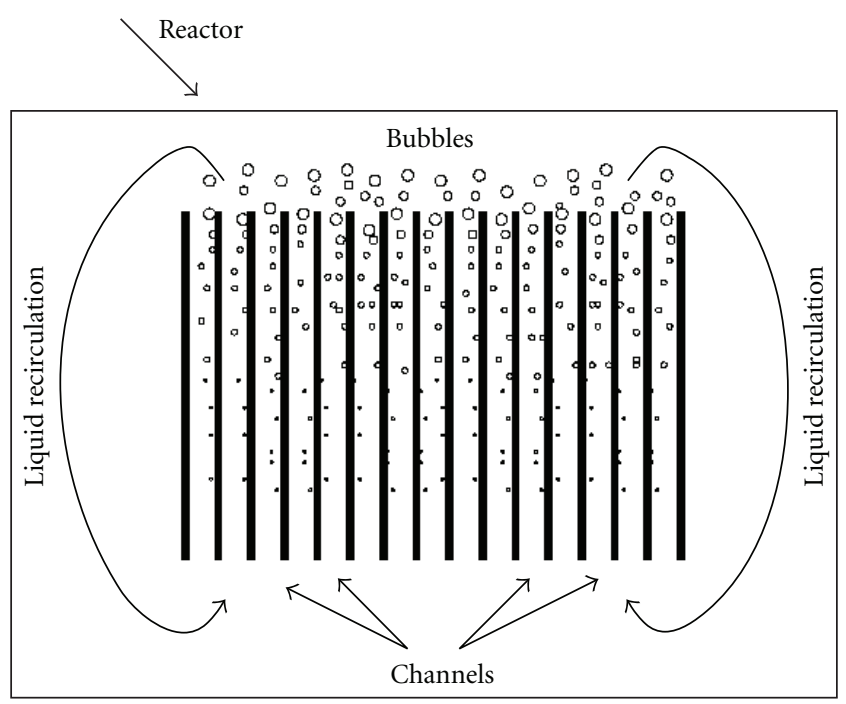

FIGURE 1: Schematic representation of an electrochemical process. A series of plates (the electrodes) are immersed in a larger container (the reactor).

can be significantly different. The extent of the difference depends to a large extent on the current density employed which governs the amount of gas generated and on the coalescence properties of the solution. Above a certain critical current density, in a noncoalescing system, where the bubbles stay small, the flow in the single-channel exhibits a transition from steady laminar to pseudoturbulent regime [9] characterized by vortices and recirculation regions. In the multichannel geometry, on the other hand, the flow does not experience this transition and remains in what we call "quasi-steady" regime. As discussed later on, this difference depends on the fact that the multichannel geometry is usually of the submerged type. The channels, in fact, are completely immersed in the liquid and, therefore, there is a common liquid region above the channels. The calculations performed in this study refer to the specific case of chlorate cells, but similar conclusions can be drawn for different systems [9].

\section{The Multichannel and the Single-Channel Systems}

We compare the flow pattern, calculated by means of CFD modeling, in the case of the single-channel (Geometry 1depicted on the left of Figure 2) and the triple-channel (Geometry 2 shown on the right of Figure 2). These channels are sometimes called "gaps" in electrochemical applications.

In both cases, hydrogen is produced at the cathode according to the reaction

$$
2 \mathrm{H}_{2} \mathrm{O}+2 \mathrm{e}^{-} \longrightarrow \mathrm{H}_{2}+2 \mathrm{OH}^{-} .
$$

Oxygen or any other gas production at the anode is ignored. The volume of $\mathrm{O}_{2}$ produced in chlorate processes is, in fact, considerably smaller $(2-4 \%)$ than that of $\mathrm{H}_{2}$, while the chlorine produced reacts rapidly with water and is not noticeable as bubbles [3].
2.1. Single-Channel (Geometry 1). In Figure 2 (left), the geometrical configuration of the single-channel is shown. The distance between the two electrodes is $h=3 \mathrm{~mm}$, the channel height $L=90 \mathrm{~mm}$. The cathode is located on the right wall in the L section at $x=h$, while the anode is located at $x=0$. An inert section of length $A=20 \mathrm{~mm}$ is placed between the hydrogen evolving wall and the top. This inert section is often present in laboratory devices but it may not be present in some industrial applications. Our objective is to identify the parameters that determine the flow filed in the active part of the channel, that is, the part in which the wall at $x=h$ is an active cathode. Some additional simulations with other channel lengths (50, 80 , and $100 \mathrm{~mm}$ ) or with a supplementary inert section at the bottom of the channel $(10 \mathrm{~mm})$ were carried out in order to verify that the flow pattern in the active part of the single-channel is not affected by these parameters. We consider only a batch electrochemical cell that contains a confined finite volume of liquid. The bottom of the channel is a solid wall and the top corresponds to the liquid-free surface.

2.2. Triple-Channel (Geometry 2). Three channels in parallel joined together by a common bottom and top section (see Figure 2 on the right) are used to represent a simplified version of the multichannel geometry. In this case, the three gaps have the same width $h=3 \mathrm{~mm}$ and a length $L$ of $90 \mathrm{~mm}$. The width of each wall is $1 \mathrm{~mm}$. The length of the upper and bottom parts are $A=20 \mathrm{~mm}$ and $B=10 \mathrm{~mm}$, respectively. The cathode, where hydrogen is produced according to (1) is located at $x=7 \mathrm{~mm}$ (two channel widths plus one wall thickness), The whole system is enclosed within an external container, which represents the reactor. The reactor is open at the top. Thus, the lateral and bottom borders are walls, while the top represents the free-liquid surface.

In both cases, the motion of the liquid is exclusively due to the momentum exchange with the rising bubbles. We assume that the physical properties of the gas are those of hydrogen, while the liquid is represented by a highly concentrated aqueous solution (see numerical values for density and viscosity in Section 5). These conditions are chosen having in mind the specific case of chlorate cells, but they can be easily changed to reflect another system. The current density at the electrodes is, in both cases, fixed to $i=500 \mathrm{~A} / \mathrm{m}^{2}$. Additionally, some simulations at lower and higher current densities are also carried out in order to better illustrate the reasons for the differences in the flow patterns observed in Geometry 1 and Geometry 2 as discussed later on. The current density is related to the gas production at the electrode, by Faraday's law

$$
j_{\text {gas }}=\frac{i M}{z F \rho_{g}},
$$

where $i\left(\mathrm{~A} / \mathrm{m}^{2}\right)$ is the current density, $M$ is the hydrogen molecular mass $\left(2 \cdot 10^{-3} \mathrm{~kg} \mathrm{~mol}^{-1}\right), z$ is the number of electrons involved in the electrochemical reaction $(z=2$, according to (1), $F$ is the Faraday constant, $\rho_{g}\left(\mathrm{~kg} \mathrm{~m}^{3}\right)$ is the gas density, and $j_{\text {gas }}(\mathrm{m} / \mathrm{s})$ is the gas superficial velocity at the electrode. The reduction of current density at 


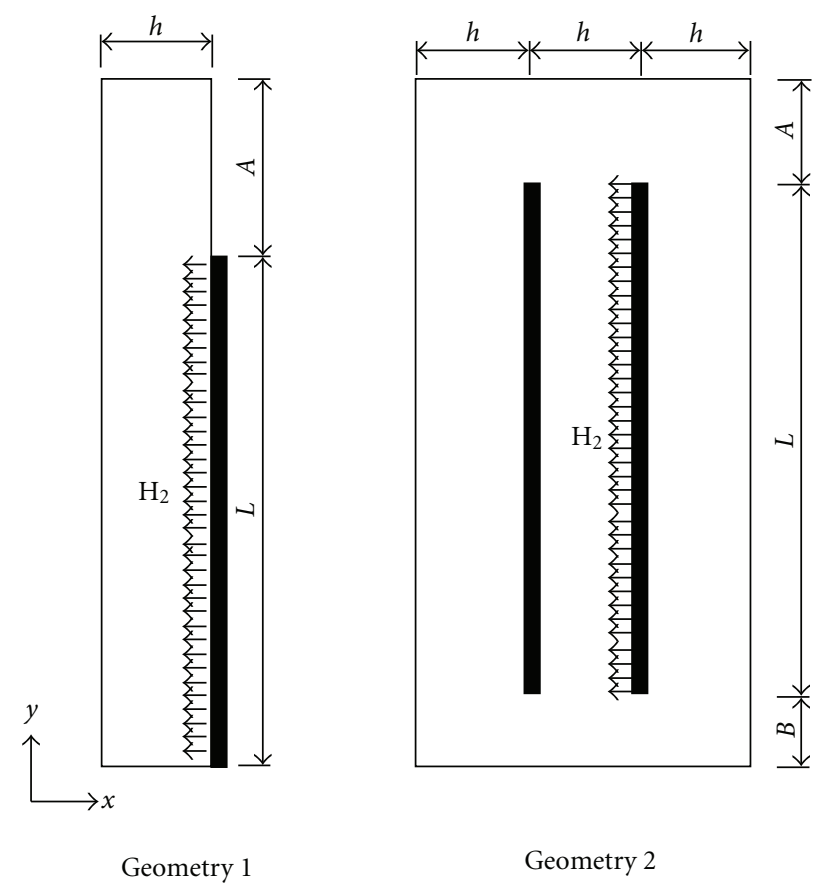

FIGURE 2: Single-channel geometry (Geometry 1) and triple-channel geometry (Geometry 2).

the electrode due to the void fraction, sometimes described by the Bruggemann equation [10], is here ignored.

Some supplementary simulations in Geometry 2 with $L=50 \mathrm{~mm}$ and an inert section of $20 \mathrm{~mm}$ (the same as in Geometry 1) were carried out in order to verify that the flow pattern in the multi-channel is not affected by these parameters. For simplicity, $\mathrm{H}_{2}$ is generated only from one side of the electrode (Figure 2 on the right). Another simulation was carried out with $\mathrm{H}_{2}$ produced on both sides. The flow in the third channel and on the top of the reactor is clearly different in this case, but the conclusions concerning the transition between the two flow patterns (Section 5.3) remain the same. We chose to discuss an electrode with only one active side on which hydrogen is produced because, in this way, a more direct comparison with the single-channel is possible. In general, when certain simplifications in the geometry or in the model were possible, we always choose the option that would reduce the difference between the flow patterns in the two geometries. In this way, we have more confidence that the observed differences between the computed flow patterns are real.

\section{Modeling}

The model for the flow in the gap is based on the following assumptions (details are given in [9]). The fluid is incompressible and Newtonian. The flow field is assumed to be two-dimensional. Temperature and all the physical properties of the channel material and fluid are constant. The bubble diameter is small and the bubbles can be treated as solid spherical particles. (We have already shown that small bubbles are the cause of flow regime transition [9] in a single-channel and in this study we examine their effect in the multiple channel geometry. The effect of coalescence is the subject of a separate study). In the channel, there is no bubble coalescence or breakup and the bubble size can be considered approximately constant. This last statement deserves additional clarification because it does not always represent reality well. In the region near the cathode, where the void fraction is higher, a variable degree of coalescence is expected. The extent of this coalescence strongly depends on the types of the electrolytes dissolved in water. Ample experimental evidence exists that certain anion/cation couples prevent bubble coalescence in water solutions [11]. Thus, according to the type, distribution, and interaction of the various ions in an electrochemical system, coalescence can be suppressed considerably, partly or not at all. In chlorate cells experiments [2,3], for instance, it was observed that a certain number of bubbles do not coalesce and maintain their initial small size. In this case, an average bubble diameter of the order of $0.01 \mathrm{~mm}$ is a reasonable approximation and some CFD studies [7] adopted this value for their calculations. One can account for the bubble coalescence in the future, but our focus here is to determine whether differences can occur between single-and multichannel flows. Our previous studies [9] indicate that presence of a sufficient number of small bubbles is needed to trigger transition in flow regime in single-channels and we investigate here whether this is the case in multiple-channels also. Thus, the bubble size in our simulations is arbitrarily fixed to $0.01 \mathrm{~mm}$. The gas production at the electrode is determined directly by (2).

The velocity field is computed by solving the continuity and Navier-Stokes equations for a biphasic flow consisting of a dispersed and continuous phase according to the so-called Euler-Euler model. In this approach, both the continuous and dispersed phases are considered to be interpenetrating 
continua and the macroscopic description of both phases is derived by averaging the conservation equations for each phase (see, e.g., [12]).

Dispersed phase:

$$
\begin{aligned}
& \frac{\partial \alpha \rho_{g}}{\partial t}+\nabla \cdot\left(\alpha \rho_{g} \mathbf{v}_{g}\right)=0, \\
& \begin{aligned}
\frac{\partial \alpha \rho_{g} \mathbf{v}_{g}}{\partial t}+ & \mathbf{v}_{g} \cdot \nabla\left(\alpha \rho_{g} \mathbf{v}_{g}\right)=\alpha\left(\mathbf{g} \rho_{g}-\nabla p\right) \\
& +\nabla \cdot\left[\alpha \mu_{g}\left(\nabla \mathbf{v}_{g}+\nabla \mathbf{v}_{g}^{T}\right)\right]-\mathbf{M} .
\end{aligned}
\end{aligned}
$$

Continuous phase:

$$
\begin{aligned}
& \frac{\partial(1-\alpha) \rho_{\ell}}{\partial t}+\nabla \cdot\left((1-\alpha) \rho_{\ell} \mathbf{v}_{\ell}\right)=0 \\
& \begin{aligned}
\frac{\partial(1-\alpha) \rho_{\ell} \mathbf{v}_{\ell}}{\partial t} & +\mathbf{v}_{\ell} \cdot \nabla \mathbf{v}_{\ell}\left[(1-\alpha) \rho_{\ell}\right] \\
= & (1-\alpha)\left(\mathbf{g} \rho_{\ell}-\nabla p\right) \\
& +\nabla \cdot\left[(1-\alpha) \mu_{\ell}\left(\nabla \mathbf{v}_{\ell}+\nabla \mathbf{v}_{\ell}^{T}\right)\right]+\mathbf{M} .
\end{aligned}
\end{aligned}
$$

The last term $\mathbf{M}$ in (4) and (6) represents the interfacial momentum transfer, which is usually divided into several different parts each contributing drag, lift, virtual mass forces, interfacial pressure, and so forth (see, e.g., [13]). In our case, only drag is considered (Wen and Yu model [14]). The effect of the lift force was evaluated [9] and resulted in little or no difference in the flow pattern. The possible presence of a slip velocity at the cathode has been discussed in [15]. The effect of bubble collision, which potentially could lead to a more spread layer of gas above the electrode, was here not considered. According to our previous investigations, however, (see $[9,16])$ the thickness of this layer should not play a major role on the stability of the flow and, therefore, on the transition between the quasisteady and the pseudoturbulent regime.

\section{Boundary Conditions}

Most of the boundaries in both Geometry 1 and Geometry 2 are simply solid walls and at these no slip boundary conditions are assigned for gas and liquid velocities. The only exceptions are the boundary conditions for the gasphase at the cathode and at the top which represents the free liquid surface. These boundary conditions are hereafter briefly discussed; more details are given in $[9,16]$.

4.1. Cathode (Gas-Phase). Concerning the liquid phase, the cathode can be viewed as a wall with no-slip conditions. This is a reasonable approximation, although, in this case, not as obvious as it might appear at first sight (more detailed discussion is given in [9]). Concerning the gas, the overall gas mass flux (i.e., the product of the gas velocity and density) at the electrode is known from (2). It is also know that

$$
j_{\text {gas }}=\alpha_{E} u_{E},
$$

but the values of $\alpha_{E}$ and $u_{E}$ are not known separately. The evaluation of both $\alpha_{E}$ and $u_{E}$ at the boundary is a recurrent issue in the Euler-Euler model. In our previous works [9, 15, 16] we showed that the void fraction at the electrode plays a role only in a region very close to the electrode, while the flow and voidage pattern in the rest of the channel are left practically unaffected by the choice of $\alpha_{E}$ and $u_{E}$, provided that their product gives the correct value of $j_{\text {gas }}$. The value of $\alpha_{E}=0.5$ was used for numerical efficiency while the value of $u_{E}$ is calculated from $(7)$. (See $[9,15]$ for the sensitivity of the results to these parameters.)

4.2. Free-Liquid Surface (Top). At the top free surface, the pressure is always fixed and a portion of the gas, as dictated by the continuity equation, is allowed to escape. Mathematically, this means that the axial gradients of both the gas velocity and the void fraction are set to zero. Concerning the continuous phase, the top is a free-slip surface, as it should be in the case of a gas-liquid interface. In one of our previous works [9], we tested various types of boundary conditions commonly employed in CFD studies at the top of bubble columns or air-lift reactors. We found that, if a long enough section A (see Geometry 1 in Figure 2) is employed the differences in flow pattern in the electrochemically active section L are minimal. For each of the above boundary condition tested at the free surface the ascending liquid "turns around" at the free surface once it reaches the top. This behavior is expected on physical grounds since the liquid cannot escape the system and, thus, it must recirculate at the top. This creates, in the single-channel, a recirculation cell, which, as explained later, is the main reason for the different behavior of the flow in Geometry 1 and Geometry 2.

\section{Results and Discussion}

A number of simulations at $\mu_{\ell}=3 \cdot 10^{-3} \mathrm{~kg} \mathrm{~m}^{-1} \mathrm{~s}^{-1}, \mu_{g}=$ $8.76 \cdot 10^{-6} \mathrm{~kg} \mathrm{~m}^{-1} \mathrm{~s}^{-1} \rho \ell=1500 \mathrm{~kg} \mathrm{~m}^{-3}, \rho_{g}=0.07 \mathrm{~kg} \mathrm{~m}^{-3} d=$ $10^{-5} \mathrm{~m}, z=2, M=2 \cdot 10^{-3} \mathrm{~kg} \mathrm{~mol}^{-1}, i=500 \mathrm{~A} \mathrm{~m}^{-2}$, and $h=3 \cdot 10^{-3} \mathrm{~m}$ were carried out. As already mentioned, the numerical values were chosen having in mind the specific case of a chlorate cell, but the results can be extended to other electrochemical applications. The mesh used is a structured rectangular mesh. It was found [9] that a mesh with a characteristic size of $0.1 \times 0.1 \mathrm{~mm}$, except for the last three cells close to the electrode, which are further divided into 15 smaller cells, is appropriate. Grid independence of the results was checked [9]. The time steps were assumed variable in order to assure a maximum Courant number smaller than 0.5 . The overall simulated time was 80 s for both configurations. Time averages were computed considering times from 40 s to 80 s, after verifying that steady state (in statistical sense) is reached. The OpenFOAM (open field operation and manipulation) CFD Toolbox was used to solve (2)-(5) and relevant boundary conditions (see [9] for other numerical details). As already stated, all the physical properties (gas and liquid densities and viscosities, bubble size, etc.), operational variables (current density, wall void fraction, etc.) and numerical parameters (mesh structure and characteristic size, strategy for determining the variable time step, etc.) are the same in the two cases. The only 
significant difference is, therefore, the presence of only one gap instead of three and the presence of the external reactor volume in Geometry 2. In the rest of the article, we show that this difference, alone, is responsible for a completely different flow pattern in the system. The interested reader is referred to our previous publications (e.g., $[9,15,16])$ for (i) the consequences of different values of $\mu_{\ell}, \rho_{\ell}, d, i, h$ on the results, (ii) the sensitivity of the flow to the boundary conditions, (iii) the discussion concerning drag and lift coefficients, (iv) a more extensive analysis of the transition between quasi-steady and pseudo-turbulent regimes in a single-channel, (v) grid accuracy, and (vi) the numerical schemes employed.

5.1. The Flow in the Three-Channel Geometry. We begin by describing the flow pattern in the multichannel configuration, because in this configuration the resulting flow is simpler. Figure 3 (dotted line) displays the void fraction profile in the middle of the central channel active height. In the two other channels, the void fraction is considerably smaller. Most of the bubbles, in fact, are either discharged at the top or trapped in the recirculation vortices at the free surface and only a small fraction can reach the two lateral channels. Figure 3 (dotted line) shows that the gas is concentrated in a region near the gas generating electrode, where the void fraction reaches very high values. This is a frequent situation in electrochemical cells and it is known as "gas blanket" formation. The model predicts the existence of this high voidage layer, but a more detailed exploration of this layer would require a submodel at the electrode. The voidage peak should probably be located directly at the electrode and not slightly shifted by $0.03 \mathrm{~mm}$ as Figure 3 indicates. This shift seems to be the result of the simplifications discussed in Section 4 . We already mentioned, however, that, except for this small region, the void fraction and velocity profiles in the rest of the channel are not affected by these simplifications (see [9] and [15] for details).

In Figure 4, a qualitative description of the flow in Geometry 2 is given, while, in Figure 5, the time-averaged velocity profiles calculated at various locations in the reactor are reported. The locations at which the velocities are displayed in Figure 5 are shown in Figure 4. The three channels are labeled $\mathrm{C} 1, \mathrm{C} 2$, and $\mathrm{C} 3$, while the three sections at $x=B+L$ (gap outlet), $x=B+L / 2$ (gap center), and $x=B$ (gap inlet) are called S1, S2, and S3, respectively. The notation, used in Figure 4 to identify the location of the reported profiles, specifies the channel first and then the section; C2S1, for instance, means Section 1 of channel 2.

The liquid motion in the channel, schematically represented in Figure 4, is driven by the gas-lift generated at the electrode, which creates a region, close to the electrode, with high upwards velocity. The rest of the liquid in the channel (gap) reacts to this high velocity sheet by moving downwards. In the central gap, therefore, it is possible to distinguish two fluid zones which move in the opposite direction. When the liquid exits from $\mathrm{C} 2$, it recirculates into $\mathrm{C} 1$ as the negative axial liquid velocities at C1S1, C1S2, and C1S3 in Figure 5 indicate. Besides the internal recirculation in $\mathrm{C} 2$, the liquid at the bottom of the reactor goes preferentially into the third

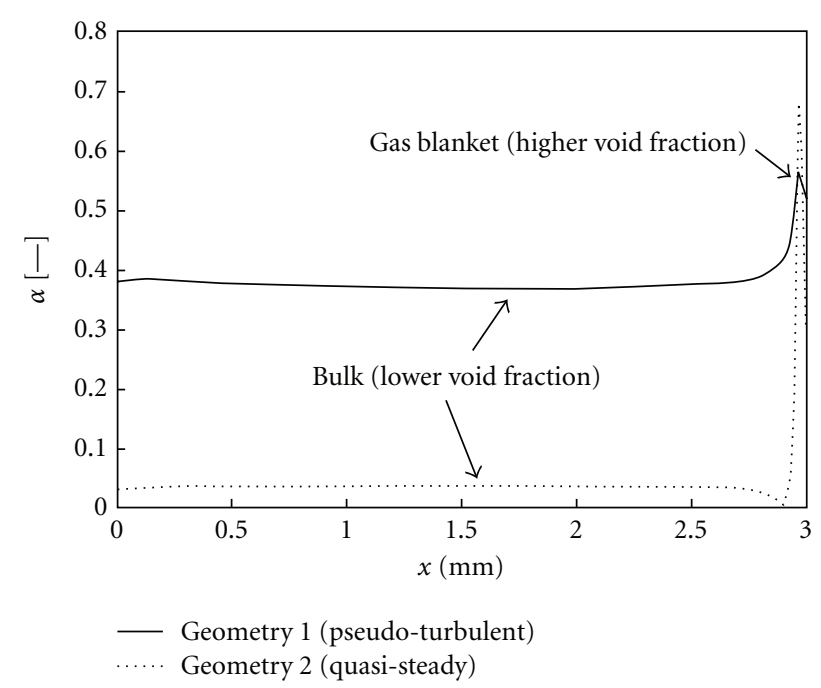

Figure 3: Comparison between the time averaged void fraction profile at the center of Geometry 1 (pseudo-turbulent regime, continuous line) and at in the center of the central channel (C2S2) of Geometry 2 (quasi-steady regime, dotted line).

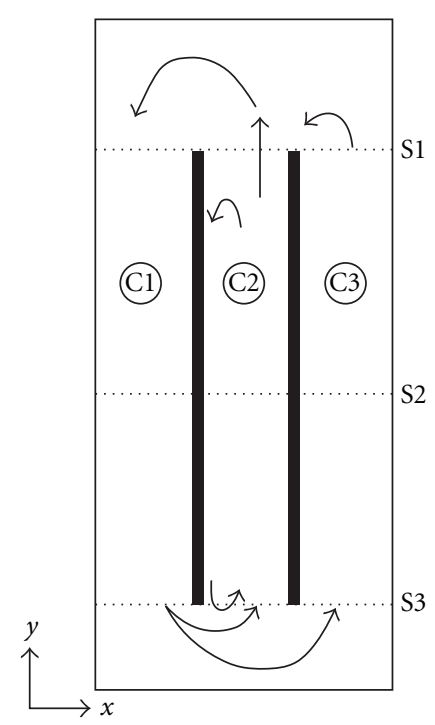

FIgURE 4: Schematic representation of the flow in Geometry 2.

channel rather than the first, as the positive velocities, in Figure 6 (C3S1, C3S2, C3S3), indicate. The fact that the flow in C3 is weakly upwards, rather than downwards as in C1, creates an overall asymmetry in the flow, which has been observed experimentally [2]. This circumstance, however, is probably of little importance for industrial configurations, where the gas is generated at the walls of every channel instead of exclusively in C2 as in this study.

5.2. The Flow in the Single-Channel Geometry. When the simulations are repeated at the same conditions for Geometry 1 , the results show a completely different picture. Figure 6 is a snapshot of the instantaneous velocity pattern in Geometry 1. The flow is highly unsteady consisting of 

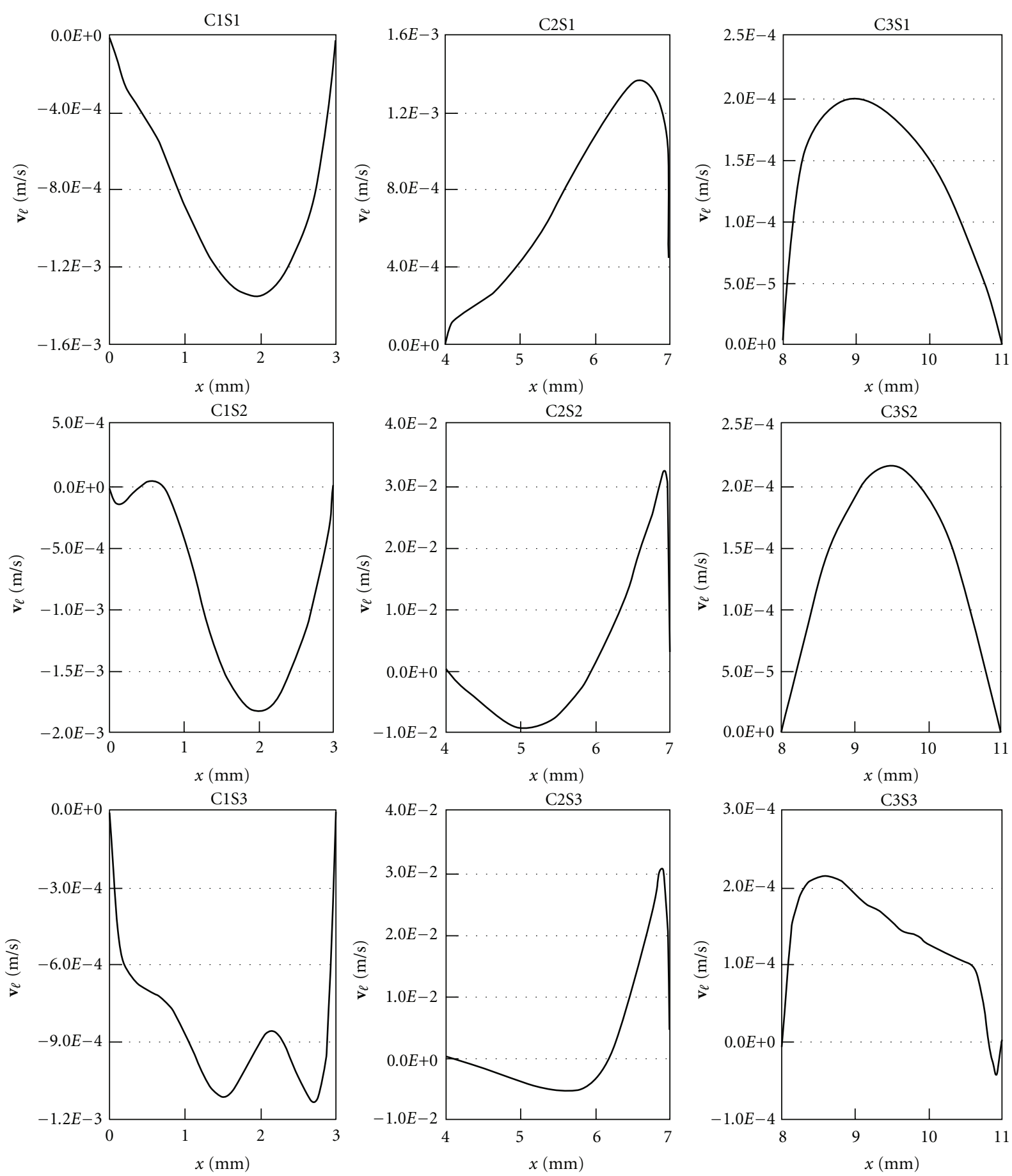

FIGURE 5: Time-averaged profiles of the vertical liquid velocity at various location of Geometry 2.

vortices and recirculation regions. There is a regular flow pattern of vortices with the same characteristic length as the channel width, whose direction of rotation alternates between clockwise and counterclockwise. It is important to note that these structures are not due to the effect of turbulence since the flow is in the laminar regime having, due to the very small bubbles' size and hydrogen density, characteristic particle Reynolds number of the order of $10^{-7}$. These fluid structures increase dramatically the liquid mixing in the gap. Figure 3 (continuous line) shows the time-averaged void fraction profile in the middle of the channel $(y=25 \mathrm{~mm})$. If we compare, in Figure 3, the continuous (Geometry 1) and dotted (Geometry 2) curves, it is immediately clear that the more intense mixing in Geometry 1 creates a more uniform void fraction profile. In comparison to the multichannel case, the voidage peak near the electrode wall has a lower value, while the gas volume fraction increases in the bulk of the channel. 


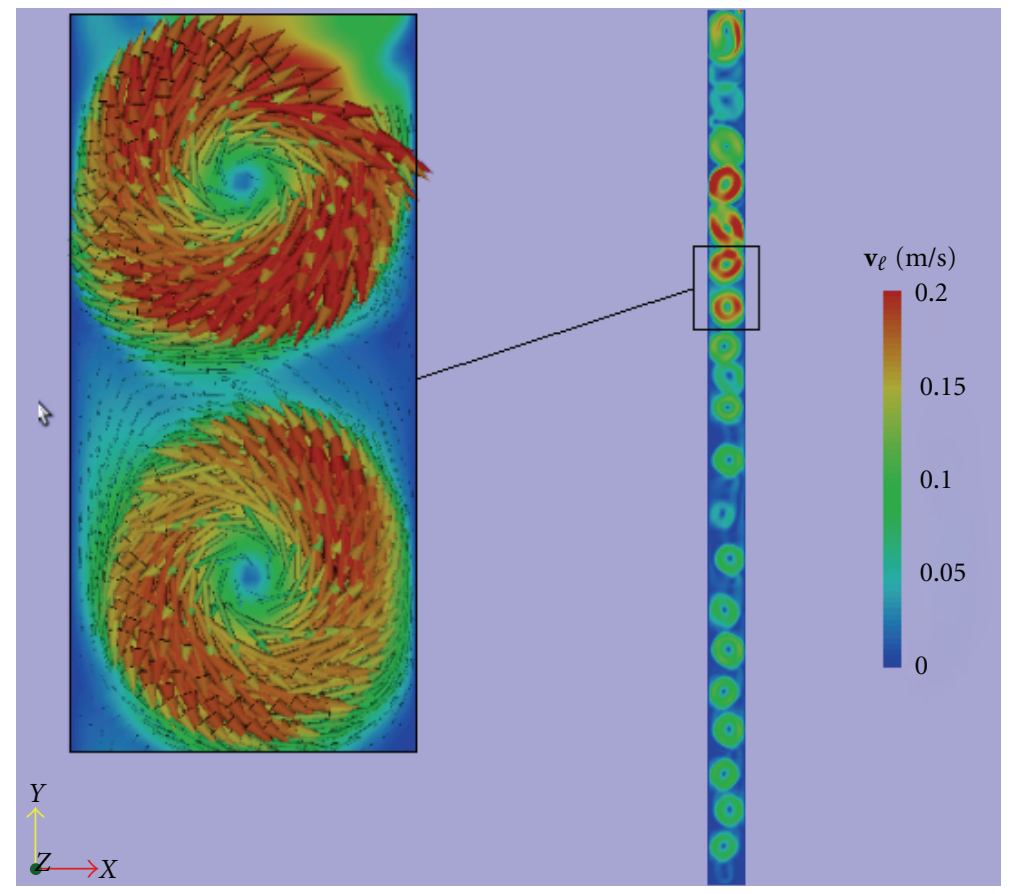

FIGURE 6: Instantaneous liquid velocity magnitude in Geometry 1 (vector representation in detail).

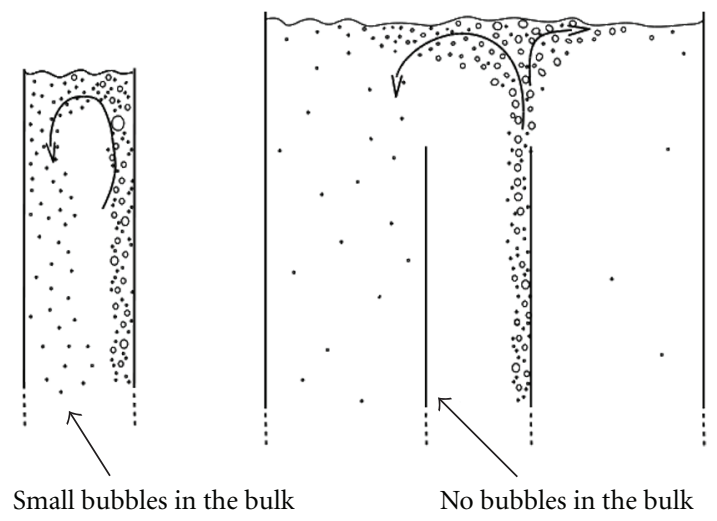

FIGURE 7: Schematic recirculation of small bubbles in single-channel (left) and multichannel (right) systems.

It is important to note that this pseudo-turbulent pattern depends on the gas-generation rate. An additional series of simulations was carried out at various value of current density $i$. If the value of this parameter is lower than $200 \mathrm{~A} / \mathrm{m}^{2}$ for the case studied here, the regime in the singlechannel is similar to the one observed in the multichannel system (quasi-steady regime). Systematic investigation of the transition between pseudo-turbulent and quasi-steady regimes is given in [15].

\subsection{The Reasons for the Difference in Single-and Multichannel} Flows. At low current densities, the only observed difference in the single-and multiple channel systems concerns the flow at the top of the channel. In the case of a single-channel, the top corresponds to the liquid free surface. In the case of a three-channel system, the free-surface is located above the channels (see Figure 2), while the section at the end of each single-channel (section S1 in Figure 4) does not represent any real physical boundary. At the free surface, the continuous phase must turn around since the liquid is entirely contained within this boundary and it cannot exit. If the diameter of the bubbles is small, the bubbles tend to follow the liquid flow due to the fact that the relative velocity is very low. In the case of a single-channel, the recirculating flow around the free-surface drags some of the bubbles back in the channel (see Figure 7 on the left). In the multichannel case, we also observe this kind of recirculation at the free-surface, but, in this geometry, the free-surface does not coincide with the top of the channel. As a consequence the bubbles are dragged back into the region above the three channels of Geometry 2 and not into the channel itself (see Figure 7 on the right). We observe the transition to the pseudo-turbulent 
regime when the void fraction in the channel bulk reaches a certain critical value. In the single-channel, due to the recirculation at the top, the bubbles tend to accumulate. If we increase the current density above a certain value (around $200 \mathrm{~A} / \mathrm{m}^{2}$ ), the flow becomes pseudo-turbulent. In the multichannel system, however, the bubbles are never accumulated at the top of a single-channel, but in the region above them; in fact, pseudoturbulence was never observed.

As shown in Figure 4, there is also global recirculation in the system. In order to determine the effect of this global recirculation on the flow pattern, we calculated the flow in Geometry 2 with $B=0$ (see Figure 2). In this case, section $\mathrm{C} 2 \mathrm{~S} 3$ is just a wall and no flow can go through it. The flow pattern, however, is not particularly affected by this change and the system remains in the quasi-steady regime. If, on the other hand, we repeat the calculation with $B \neq 0$ and $A=0$ (with the channels now separated at the top but still connected at the bottom), the transition to pseudo-turbulence occurs as in the single-channel case. We can conclude, therefore, that the main phenomena affecting the flow pattern in the system depend on the conditions at the top of the channel rather than at the bottom. As a final test, we run some single-channel simulations imposing an inlet velocity at the bottom. When this velocity reaches the value of approximately $0.1 \mathrm{~m} / \mathrm{s}$, the recirculation pattern responsible for the pseudo-turbulent regime is destroyed. This value, however, is at least one order of magnitude higher than the average inlet velocity at C2S3 (see Figure 5). Thus, the overall recirculation in the reactor does not create at the bottom of the electrochemically active channel an inlet flow large enough to prevent transition to pseudo-turbulence.

A direct experimental comparison of the two systems with the goal of highlighting the difference in the flow patterns is not available in the literature yet. There are, however, some significant clues in the reported studies that seem to confirm our hypotheses concerning the importance of the small bubbles (around $10 \mu \mathrm{m}$ ) for the aforementioned regime transition. In Boissonneau and Byrne [3], for instance, where, in a single-gap configuration, vortexes and recirculation regions were detected in the flow, the authors also observed that not all the gas was liberated from the solution and that a certain amount of small bubbles, mostly of the diameter less than $10 \mu \mathrm{m}$, was recirculated in the channel.

All these observations are in perfect agreement with a more theoretical approach we employed in one of our previous works [16] in order to investigate the transition from quasi-steady state to pseudo-turbulence in a singlechannel. The flow was assumed pseudohomogeneous and piecewise linear, and the effect of velocity and pressure disturbances on the flow stability was investigated by means of the Rayleigh equation (here, we discuss only the results; the lengthy mathematical manipulations are omitted). Our analysis shows that the fundamental parameter for the stability of the flow is the density ratio $q$ between the socalled gas-blanket (the region near the cathode where most of the gas is concentrated, see Figure 3) and the bulk (the rest of the channel where the void fraction is lower, see Figure 3). Above a certain critical $q_{c r}$, as discussed in [16], the system becomes unstable and flow disturbances with initial infinitesimal amplitude grow exponentially.

If, as a first approximation, we assume that the gasblanket is always entirely constituted of gas, the only way to increase the density ratio $q$ between the gas-blanket and the bulk is to decrease the density of the bulk. This happens, for instance, when, due to the flow recirculation at the top of the channel (see the left part of Figure 7), more bubbles are dragged back into the bulk thus reducing the apparent density of this region and increasing the ratio $q$. When the value of $q$ increases above $q_{c r}$, the flows become unstable and transition to pseudo-turbulence occurs. In the multichannel case, however, (see the right part of Figure 7) the bubbles, as we already explained, do not recirculate in the bulk. As a consequence $q$ does not increase, the flow remains stable and transition to pseudo-turbulence does not occur.

\section{Conclusions}

The flow pattern and the void fraction distribution in gasevolving electrochemical cells can be significantly different in a single-channel than in multichannel configurations as shown in this study. This is mostly due to a recirculation pattern at the top of the single-channel case that contributes to the accumulation of the smallest bubbles. While transition from quasi-steady flow pattern to pseudo-turbulent occurs in single-channel flows at sufficiently high gas production rate (i.e., at high current density) involving sufficiently small bubbles, this transition is averted in multichannel flows. This happens because the gaps in multichannel systems are usually completely immersed in the liquid. Based on the presented results, we conclude that the presence of a region external to the individual channel (gap) plays a significant role in the evolution of the hydrodynamics internal to the gap. If the goal of single-channel laboratory studies is to provide information for large multichannel setups, then appropriate care must be taken to ensure the similarity of flow regime and flow pattern and this issue must be addressed when experiments are planned. For example, if the single-channel (gap) is immersed in a larger experimental apparatus, where global recirculation is allowed, the external flow conditions would probably be similar to the multichannel case and the resulting flow patterns in individual channels comparable.

\section{Notations}

$\alpha: \quad$ The void fraction $[-]$

$\alpha_{E}$ : The void fraction at the electrode [-]

$A$ : Inert section in Geometry 1 or distance top-electrode in Geometry 2 [m]

$B: \quad$ Distance top-electrode in Geometry $2[\mathrm{~m}]$

$d$ : Bubble diameter [m]

F: $\quad$ Faraday constant $96487 \mathrm{~A} \mathrm{~s} \mathrm{~mol}^{-1}$

g: Gravity acceleration constant $9.81\left[\mathrm{~m} \mathrm{~s}^{-2}\right]$

$h$ : Distance between the two electrodes [m]

$i$ : $\quad$ Current density $\left[\mathrm{A} \mathrm{m}^{-2}\right]$

$j_{\text {gas }}$ : Inlet superficial gas velocity $\left[\mathrm{m} \mathrm{s}^{-1}\right]$

$L$ : Active channel height $[\mathrm{m}]$ 
$L_{\mathrm{TOT}}$ : Total height $(L+A)$ in Geometry 1 or $(L+A+B)$ in Geometry $2[\mathrm{~m}]$

$M: \quad$ Molecular mass $\left[\mathrm{kg} \mathrm{mol}^{-1}\right]$

$\mu_{g}: \quad$ Gas viscosity $\left[\mathrm{kg} \mathrm{m}^{-1} \mathrm{~s}^{-1}\right]$

$\mu_{l}: \quad$ Liquid viscosity $\left[\mathrm{kg} \mathrm{m}^{-1} \mathrm{~s}^{-1}\right]$

$\rho_{g}: \quad$ Gas density $\left[\mathrm{kg} \mathrm{m}^{-3}\right]$

$\rho_{l}: \quad$ Liquid density $\left[\mathrm{kg} \mathrm{m}^{-3}\right]$

$t$ : Time [s]

$u_{E}$ : Inlet gas velocity at the electrode $\left[\mathrm{m} \mathrm{s}^{-1}\right]$

$v_{g}$ : Local and instantaneous gas velocity $\left[\mathrm{m} \mathrm{s}^{-1}\right]$

$v_{l}$ : Local and instantaneous liquid velocity $\left[\mathrm{m} \mathrm{s}^{-1}\right]$

$v_{l x}: \quad$ Component of $\mathbf{v}_{\ell}$ in the $x$-direction $\left[\mathrm{m} \mathrm{s}^{-1}\right]$

$v_{l y}$ : Component of $\mathbf{v}_{\ell}$ in the $y$-direction $\left[\mathrm{m} \mathrm{s}^{-1}\right]$

$z$ : Number of electrons involved in the electrode reaction $[-]$.

\section{Acknowledgment}

This work has been performed under the MELPRIN Project founded by the CEC under the sixth Framework Program.

\section{References}

[1] H. Vogt, "Gas evolving electrodes," in Comprehensive Treatise of Electrochemistry, vol. 6, pp. 445-489, Plenum Press, New York, NY, USA, 1983.

[2] P. Hell and J. Wanngard, Private Communication, Eka Chemicals, Mannheim, Germany, 2009.

[3] P. Boissonneau and P. Byrne, "Experimental investigation of bubble-induced free convection in a small electrochemical cell," Journal of Applied Electrochemistry, vol. 30, no. 7, pp. 767-775, 2000.

[4] M. D. Mat and K. Aldas, "Application of a two-phase flow model for natural convection in an electrochemical cell," International Journal of Hydrogen Energy, vol. 30, no. 4, pp. 411-420, 2005.

[5] M. D. Mat, K. Aldas, and O. J. Ilegbusi, "A two-phase flow model for hydrogen evolution in an electrochemical cell," International Journal of Hydrogen Energy, vol. 29, no. 10, pp. 1015-1023, 2004.

[6] R. Wedin and A. A. Dahlkild, "On the transport of small bubbles under developing channel flow in a buoyant gasevolving electrochemical cell," Industrial and Engineering Chemistry Research, vol. 40, no. 23, pp. 5228-5233, 2001.

[7] H. Vogt, "The rate of gas evolution at electrodes-II. An estimate of the efficiency of gas evolution on the basis of bubble growth data," Electrochimica Acta, vol. 29, no. 2, pp. 175-180, 1984.

[8] H. Vogt, "The rate of gas evolution of electrodes-I. An estimate of the efficiency of gas evolution from the supersaturation of electrolyte adjacent to a gas-evolving electrode," Electrochimica Acta, vol. 29, no. 2, pp. 167-173, 1984.

[9] A. Alexiadis, M. P. Dudukovic, P. Ramachandrana, A. Cornell, J. Wanngard, and A. Bokkers, "Liquid-gas flow patterns in a narrow electro-chemical channel," Chemical Engineering Science, vol. 66, pp. 2252-2260, 2011.
[10] L. J. J. Janssen, "Behaviour of and mass transfer at gas-evolving electrodes," Electrochimica Acta, vol. 34, no. 2, pp. 161-169, 1989.

[11] V. S. J. Craig, "Bubble coalescence and specific-ion effects," Current Opinion in Colloid and Interface Science, vol. 9, no. 1-2, pp. 178-184, 2004.

[12] D. A. Drew, "Mathematical-modeling of 2-phase flow," Annual Review of Fluid Mechanics, vol. 15, pp. 261-291, 1983.

[13] D. Z. Zhang and A. Prosperetti, "Momentum and energy equations for disperse two-phase flows and their closure for dilute suspensions," International Journal of Multiphase Flow, vol. 23, no. 3, pp. 425-453, 1997.

[14] C. Y. Wen and Y. H. Yu, "Mechanics of fluidization," Chemical Engineering Progress Symposium Series, vol. 62, no. 62, pp. 100$111,1966$.

[15] A. Alexiadis, M. P. Dudukovic, P. Ramachandrana, A. Cornell, J. Wanngard, and A. Bokkers, "On the electrode boundary conditions in the simulation of two phase flow in electrochemical cells," International Journal of Hydrogen Energy, vol. 36, no. 14, pp. 8557-8559, 2011.

[16] A. Alexiadis, M. P. Dudukovic, P. Ramachandrana, A. Cornell, J. Wanngard, and A. Bokkers, "Transition to pseudoturbulence in a narrow gas-evolving channel," Theoretical and Computational Fluid Dynamics. In press. 

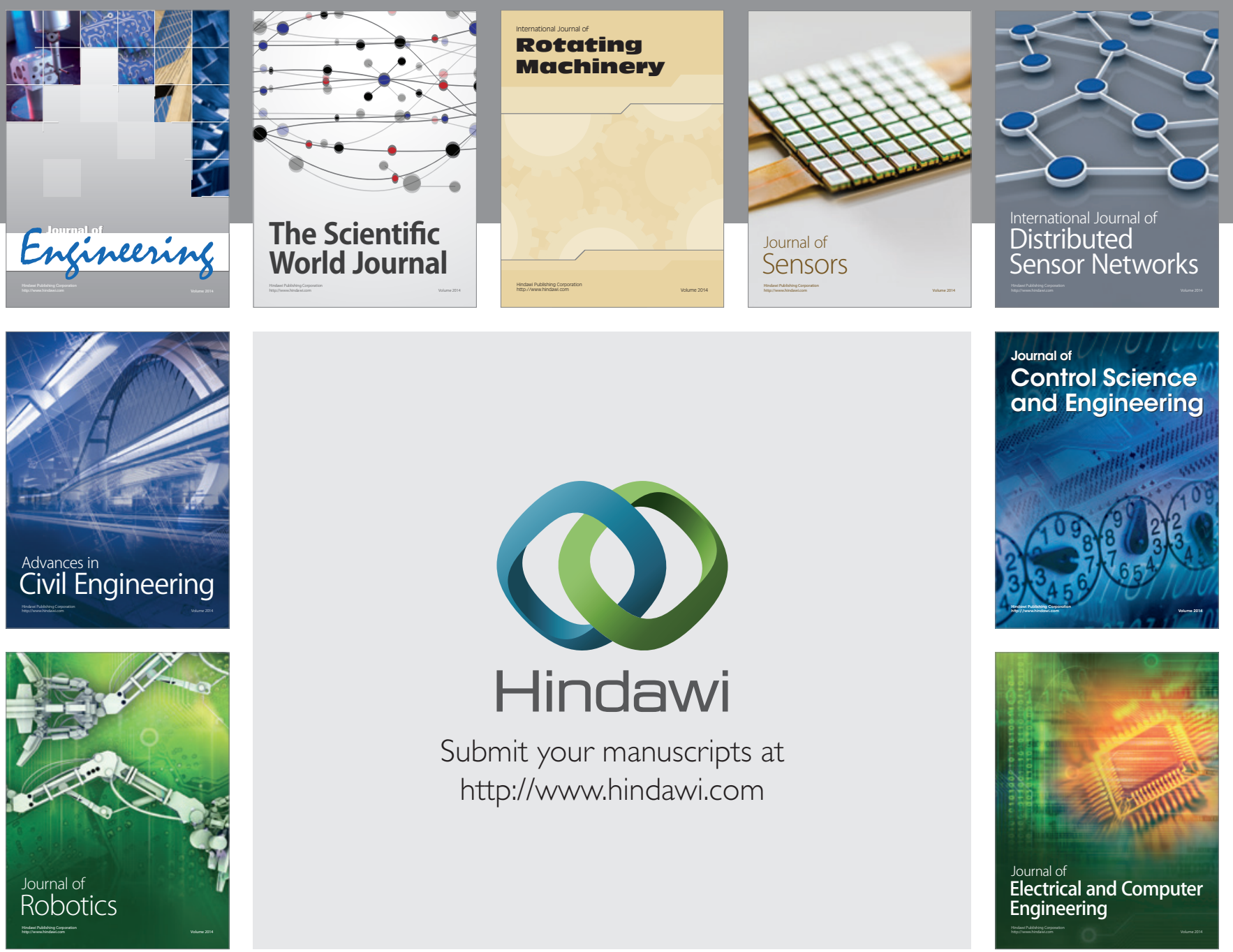

Submit your manuscripts at

http://www.hindawi.com
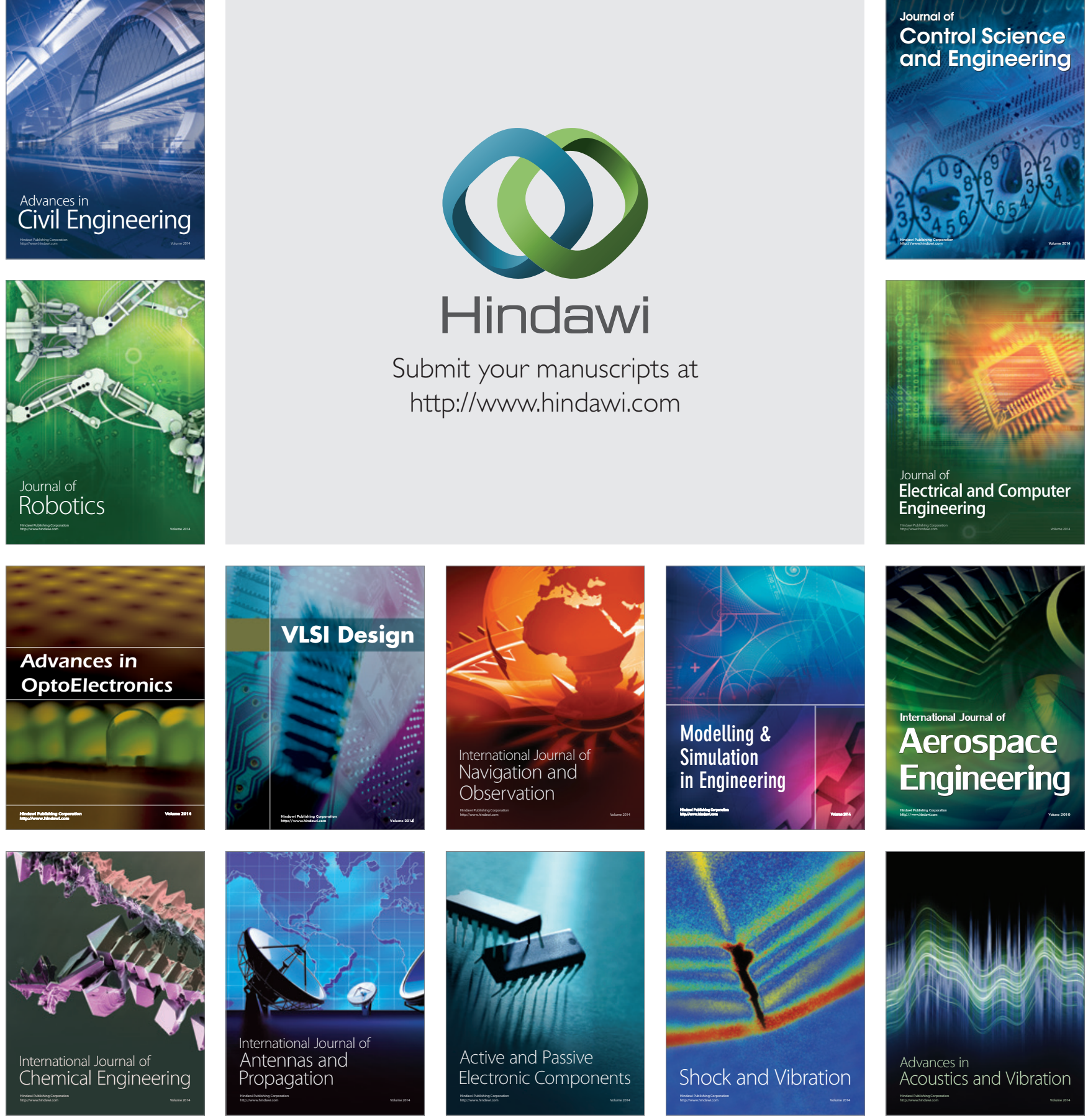